\title{
Phantom, clinical, and texture indices evaluation and optimization of a penalized-likelihood image reconstruction method (Q.Clear) on a BGO $\mathrm{PET} / \mathrm{CT}$ scanner
}

\author{
Gabriel Reynés-Llompart ${ }^{\text {a) }}$ \\ PET Unit. Nuclear Medicine Dept, IDI. Hospital U. de Bellvitge-IDIBELL, L'Hospitalet de Llobregat, Barcelona, Spain \\ Medical Physics Department, Institut Català d'Oncologia, L'Hospitalet de Llobregat, Barcelona, Spain \\ Cristina Gámez-Cenzano, José Luis Vercher-Conejero, Aida Sabaté-Llobera, and \\ Nahúm Calvo-Malvar \\ PET Unit. Nuclear Medicine Dept, IDI. Hospital U. de Bellvitge-IDIBELL, L'Hospitalet de Llobregat, Barcelona, Spain \\ Josep M Martí-Climent \\ Nuclear Medicine, Clínica Universidad de Navarra, Pamplona, Spain
}

(Received 11 December 2017; revised 2 April 2018; accepted for publication 10 May 2018; published 8 June 2018)

Introduction: The aim of this study was to evaluate the behavior of a penalized-likelihood image reconstruction method (Q.Clear) under different count statistics and lesion-to-background ratios (LBR) on a BGO scanner, in order to obtain an optimum penalization factor ( $\beta$ value) to study and optimize for different acquisition protocols and clinical goals.

Methods: Both phantom and patient images were evaluated. Data from an image quality phantom were acquired using different Lesion-to-Background ratios and acquisition times. Then, each series of the phantom was reconstructed using $\beta$ values between 50 and 500, at intervals of 50. Hot and cold contrasts were obtained, as well as background variability and contrast-to-noise ratio (CNR). Fifteen ${ }^{18} \mathrm{~F}-\mathrm{FDG}$ patients (five brain scans and 10 torso acquisitions) were acquired and reconstructed using the same $\beta$ values as in the phantom reconstructions. From each lesion in the torso acquisition, noise, contrast, and signal-to-noise ratio (SNR) were computed. Image quality was assessed by two different nuclear medicine physicians. Additionally, the behaviors of 12 different textural indices were studied over 20 different lesions.

Results: Q.Clear quantification and optimization in patient studies depends on the activity concentration as well as on the lesion size. In the studied range, an increase on $\beta$ is translated in a decrease in lesion contrast and noise. The net product is an overall increase in the SNR, presenting a tendency to a steady value similar to the CNR in phantom data. As the activity concentration or the sphere size increase the optimal $\beta$ increases, similar results are obtained from clinical data. From the subjective quality assessment, the optimal $\beta$ value for torso scans is in a range between 300 and 400, and from 100 to 200 for brain scans. For the recommended torso $\beta$ values, texture indices present coefficients of variation below $10 \%$.

Conclusions: Our phantom and patients demonstrate that improvement of CNR and SNR of Q.Clear algorithm which depends on the studied conditions and the penalization factor. Using the Q.Clear reconstruction algorithm in a BGO scanner, a $\beta$ value of 350 and 200 appears to be the optimal value for 18F-FDG oncology and brain PET/CT, respectively. () 2018 American Association of Physicists in Medicine [https://doi.org/10.1002/mp.12986]

Key words: Bayesian penalized likelihood, heterogeneity, image quality, PET/CT, Q.Clear, texture

\section{INTRODUCTION}

An accurate quantification of tracer uptake is essential to increase the reliability of Positron Emission Tomography (PET) in conjunction with Computed Tomography (CT) on many advanced applications. ${ }^{1,2}$

Standardized Uptake Values (SUV) or its variants are frequently used to provide a semiquantitative measure of tracer uptake. $^{3}$ When iterated to SUV convergence, MaximumLikelihood Expectation Maximization (MLEM) or Ordered Subset Expectation Maximization (OSEM) algorithms produce noisy images due to a problem of ill-conditioning, that is, the results have a large dependence on small changes in the initial data. ${ }^{4,5}$ Consequently, the iteration process must be stopped prior to an ideal SUV convergence, ${ }^{6}$ understood as the solution of the iterative process closest to the real SUV, which is also a factor to include in the criticism of SUV quantification. $^{7}$

A possible solution to the ill-conditioning problem is adding an edge-preserving penalty term in the reconstruction process. Following this approach, the Q.Clear algorithm (GE Healthcare, Milwakee) uses a block sequential regularized expectation maximization (BSREM) method for reconstruction, which includes a point-spread function (PSF) modeling 
and the control of noise through the use of a penalty term. ${ }^{8}$ The penalty term imposes more smoothing in lower activity regions and less smoothing in higher activity regions or in the neighborhood of high intensity edges, resulting in smoother cold backgrounds and improved hot lesions signalto-noise ratio. ${ }^{9}$ At the same time, the use of a penalty function allows an effective SUV convergence, providing more accurate values. $^{9-13}$ On its commercial implementation, the behavior of the Q.Clear reconstruction is controlled by a single parameter, called $\beta$, which determines the strength of the penalty function.

On clinical practice, different PET applications have different count statistics. The Q.Clear algorithm has a dependence on the region activity, allowing a window to optimize it depending on each application, as could be torso ${ }^{18} \mathrm{~F}-\mathrm{FDG}$ protocols for oncological scans, brain studies or other specific imaging goals.

Q.Clear algorithm has been first introduced on Lutetiumyttrium oxyorthosilicate (LYSO) PET systems and its performance has been reported, ${ }^{11}$ though it has not been evaluated in the new generation of Bismuth Germanate Oxide (BGO) PET scanners. These new scanners do not incorporate timeof-flight (TOF) but present higher sensitivity than their predecessors. ${ }^{14}$

TOF increases the signal-to-noise ratio (SNR) of the image, this could be compensated in the Discovery IQ via the gain on the sensibility, despite that, there is an intrinsic difference on the image produced by both equips. ${ }^{15}$ Hence there is not a clear a priory relation between the scanner type and the choice of the reconstruction parameter. A bad optimization of the $\beta$ value could be translated to an over-smoothing of the images, which could have its impact especially on the detection of small lesions.

For a general oncological case in a LYSO scanner and for ${ }^{18} \mathrm{~F}$-FDG, the recommended $\beta$ value of 400 is the most commonly used, ${ }^{11,12,16}$ although this value assumes a general case without optimizing different count statistics or ratios. Moreover, all previously reported studies concerning Q.Clear behavior were in the base of the impact on SUV related metrics. However, there is an ongoing interest in alternative measurements such as the metabolic tumor volume (MTV) or heterogeneity metrics, measured as textural indices (TI), ${ }^{17}$ and its dependence on Q.Clear optimization is not yet studied.

The aim of this study was to evaluate the behavior of Q.Clear algorithm under different counts statistics and lesionto-background ratios (LBR) on a BGO scanner. Data were used to study and optimize different acquisition protocols and clinical applications, and its impact on different quantitative metrics, including textural indices.

\section{METHODS}

\section{A. Discovery IQ PET/CT}

The Discovery IQ with 5 rings (D-IQ-5) system (General Electric Healthcare (GEH), Milwaukee, WI, USA) combines a BGO-based PET tomograph with a 16-slice CT scanner. Scanner performance has been previously described. ${ }^{18}$ In short, the D-IQ-5 PET enables an axial and transaxial physical field of view (FOV) of 26 and $70 \mathrm{~cm}$ respectively, with 79 image planes and $3.27 \mathrm{~mm}$ plane spacing. The system includes the Q.Clear reconstruction, and two OSEM iterative reconstruction algorithms, marketed as VUE Point HD (VPHD), and the VPHD with PSF correction (VPHD-S).

\section{B. Phantom evaluation}

A National Electrical Manufacturers Association (NEMA) body image quality phantom ${ }^{19}$ was used, consisting of six spheres with diameters of 10, 13, 17, 22, 28, and $37 \mathrm{~mm}$. The phantom was filled with a target initial radioactivity concentration of $5.5 \mathrm{kBq} / \mathrm{cm}^{3}{ }^{3}$ The four smaller spheres were filled with three LBR of $2: 1,4: 1$, and 8:1. The remaining spheres were filled with nonradioactive water. The body phantom was positioned with all spheres aligned within the same transaxial image plane in the center of the FOV. For each ratio, three sequential list mode measurements of $350 \mathrm{~s}$ were acquired for a single-bed position. For each acquisition, the phantom was reconstructed using $\beta$ values between 50 and 500 , at intervals of 50, and acquisition times (in parenthesis total million counts) of $15 \mathrm{~s}(19 \pm 4), 45 \mathrm{~s}(56 \pm 9), 60 \mathrm{~s}$ $(72 \pm 12), 120 \mathrm{~s}(140 \pm 30), 180 \mathrm{~s}(230 \pm 50)$, and $350 \mathrm{~s}$ $(360 \pm 10)$, in order to obtain different count statistics as a simulation of lower activity acquisitions or body regions with different activity concentrations A total of 150 image sets were obtained, using a $60 \mathrm{~cm}$ FOV reconstruction, and a $256 \times 256$ matrix size (transverse pixel size of $2.3 \mathrm{~mm}$ ).

In order to evaluate image quality, different figures of merit were used. Contrast recovery (CR) coefficients were defined as NEMA NU-2-2012, ${ }^{19}$ including hot percent contrast recovery for each hot sphere (HCR), cold percent contrast recovery for each cold sphere (CCR) and background variability $(\mathrm{BV})$ for all spheres. The previous parameters were also compared to a standard OSEM + PSF reconstruction (VPHD-S with 12 subsets, 4 iterations, and a $4.8 \mathrm{~mm}$ FWHM filtering); previously optimized to fulfill clinical needs. Additionally, the CNR for each sphere ${ }^{11}$ was used. A detailed description of each parameter can be found in the supplemental material.

\section{C. Clinical evaluation}

Two different types of studies were selected: brain acquisitions (neuro-oncological) and torso acquisitions (oncological whole body acquisition excluding legs and head). For both scan types, 10 patients were selected. Informed consent is not necessary for retrospective reviews of this nature in our institution. Torso acquisitions were selected when presented at least one visible lesion, while brain acquisitions were selected from nontumoral patient scans. All patients were injected with $2.5 \pm 0.2 \mathrm{MBq} / \mathrm{kg}$ and rested during $60 \pm 10 \mathrm{~min}$. Acquisition times were $2 \mathrm{~min} / \mathrm{bed}$ for torso and $5 \mathrm{~min} / \mathrm{bed}$ for brain studies. Brain exams were reconstructed with a 
$40 \mathrm{~cm}$ FOV and torso exams with a $60 \mathrm{~cm}$ FOV, both with a matrix of $256 \times 256$. For each study, reconstructions with different $\beta$ values between 50 and 500 at intervals of 50 were done. All images were also reconstructed using VPHD-S (12 subsets, 4 iterations, and a $4.8 \mathrm{~mm}$ Gaussian post-filtering).

For torso acquisitions, a single distinctive lesion from each patient was analyzed to conduct a quantitative analysis. Lesion SUV maximum $\left(\mathrm{SUV}_{\max }\right)$ and mean $\left(\mathrm{SUV}_{\text {mean }}\right)$ values were recorded, corresponding to a volume of interest (VOI) defined as the volume segmented from a threshold of $41 \%$ of the $\mathrm{SUV}_{\max }$, also corresponding to the MTV. ${ }^{3}$ Moreover, to evaluate the reconstruction parameters, the following figures of merit for each lesion were computed: SNR, contrast, and noise. ${ }^{20}$ A detailed description of each figure of merit can be found in the supplemental material.

Each set of images was evaluated by two different nuclear medicine physicians. All patients and studies were anonymized, and cases were reviewed in a randomized order, flowing two steps: first, a group of five patients were clinically evaluated in all reconstructions, and secondly five more torso patients were analyzed but just in a reduced range of reconstructions ( $\beta$ values between 300 and 450). All studies represent a total of 75 cases for torso and 55 cases for brain.

For each torso reconstruction, the reviewer was asked to rank from 0 to 4 (meaning 0 non-diagnostic and four excellent images) the general lesion conspicuity and the image quality of: liver, mediastinum, lung, bone marrow, and overall scan. For brain acquisitions, the reviewer was asked to rank with the same criteria the definition of the gyri and basal ganglia, as well as the overall image quality. The inter-rater agreement was calculated using the weighted Cohen Kappa.

\section{D. Texture indices evaluation}

Twenty lesions on 17 different patients were used to evaluate the clinical variation and robustness of each TI in relation to the Q.Clear regularization parameter. All images were reconstructed using the same interval of $\beta$ values as previous sections, although the reconstructions using $\beta=50$ were excluded due to the elevated level of noise.

Due to the vast number of TI, we focused in a subset of indices described in previous publications. ${ }^{18,19}$ From the cooccurrence matrix, the second order parameters of Homogeneity, Entropy, and Correlation were calculated. From the Gray-Level Run Length Matrix (GLRM) the Long-Run Emphasis (LRE), Short-Run Emphasis (SRE), Low GrayLevel Run Emphasis (LGRE), and High Gray-Level Run Emphasis (HGRE) were calculated. From the Gray-Level Zone Matrix (GLZM), the short-zone emphasis (SZE), LongZone Emphasis (LZE), Low-Level Zone Emphasis (LGZE), and High-Level Zone Emphasis (HGZE) were calculated.

TI were obtained with an in-house software developed in Python 3.4. Images were resampled to 64 bins, using absolute resampling between 0 and $20 .{ }^{21}$ To compute the MTV, a VOI of $40 \%$ of the $\mathrm{SUV}_{\max }$ was performed for each lesion in all reconstructions, as described earlier.
To model the robustness of image features over the different reconstructions for each patient, we calculated the $\mathrm{COV}$ as follows ${ }^{22}$ :

$$
\operatorname{COV}_{i}=100 \cdot \frac{T I_{i, S D}}{T I_{i, \text { mean }}},
$$

where $\mathrm{TI}_{\mathrm{i}, \mathrm{SD}}$ and $\mathrm{TI}_{\mathrm{i} \text {,mean }}$ refers to the standard deviation and the mean of the $\mathrm{i}^{\text {th }}$ TI over the studied range of reconstructions, respectively. Mean COV was computed and presented for each TI.

\section{RESULTS}

\section{A. Phantom evaluation}

Background phantom concentration for the 8:1, 4:1, and 2:1 LBR were $6.2,5.3$, and $5.4 \mathrm{kBq} / \mathrm{cm}^{3}$. Figure 1 shows the hot and cold $\mathrm{CR}$ against $\mathrm{BV}$ for different $\beta$ values, using the three LBR and a time acquisition of $120 \mathrm{~s}$. Results from the VPHD$\mathrm{S}$ algorithm under clinical conditions are also shown. In general terms, the $\beta$ value acts as a noise penalizing factor, decreasing $\mathrm{BV}$ as it increases in value. There is also a decrease in CR coefficients. For hot spheres, the variation depends on the LBR and sphere diameters; when one of these parameters increases, the HCR seems to approach faster to a steady value, where its dependence on the $\beta$ value decreases. For the CCR coefficients, there is also an increase when there is a decrease on the $\beta$ value, although it does not seem to depend on the LBR.

To show the dependence on LBR, sphere size and counting statistics, Fig. 2 presents the CNR as a function of the $\beta$ values, averaging one variable over the others. There is not a significant difference on the CNR behavior for $4: 1$ or $8: 1$ LBR; although in the 2:1 LBR there is a clear different pattern, as the convergence of the CNR appears at the initial $\beta$ values [Fig. 2(a)]. As the sphere size decreases [Fig. 2(b)], the CNR convergence appears earlier in the range of the studied values. For the three smallest spheres, there is a maximum followed by an observable decrease on the CNR for the highest $\beta$ values. The CNR is higher for longer acquisition times [Figs. 2(c) and 2(d)]. For larger spheres, the convergence seems to be for a $\beta$ outside the studied range $(\beta>500)$. To obtain an optimal $\beta$ value applicable to clinical practice, Table I presents the mean values of $\beta$ for different groups of hot spheres as a function of acquisition time, which maximizes the CNR. As the time increases or the size of spheres in the group decreases, the optimal $\beta$ decreases. Tables S1$\mathrm{S} 3$, present the same results as a function of each LBR.

For the phantom cold lesions, CNR also increases as the acquisition time or $\beta$ value increase (Fig. S1), almost independently from LBR.

\section{B. Clinical evaluation}

Representative examples of patient images obtained with the different reconstruction parameters, are presented in Fig. 3 for the case of torso and brain studies. 


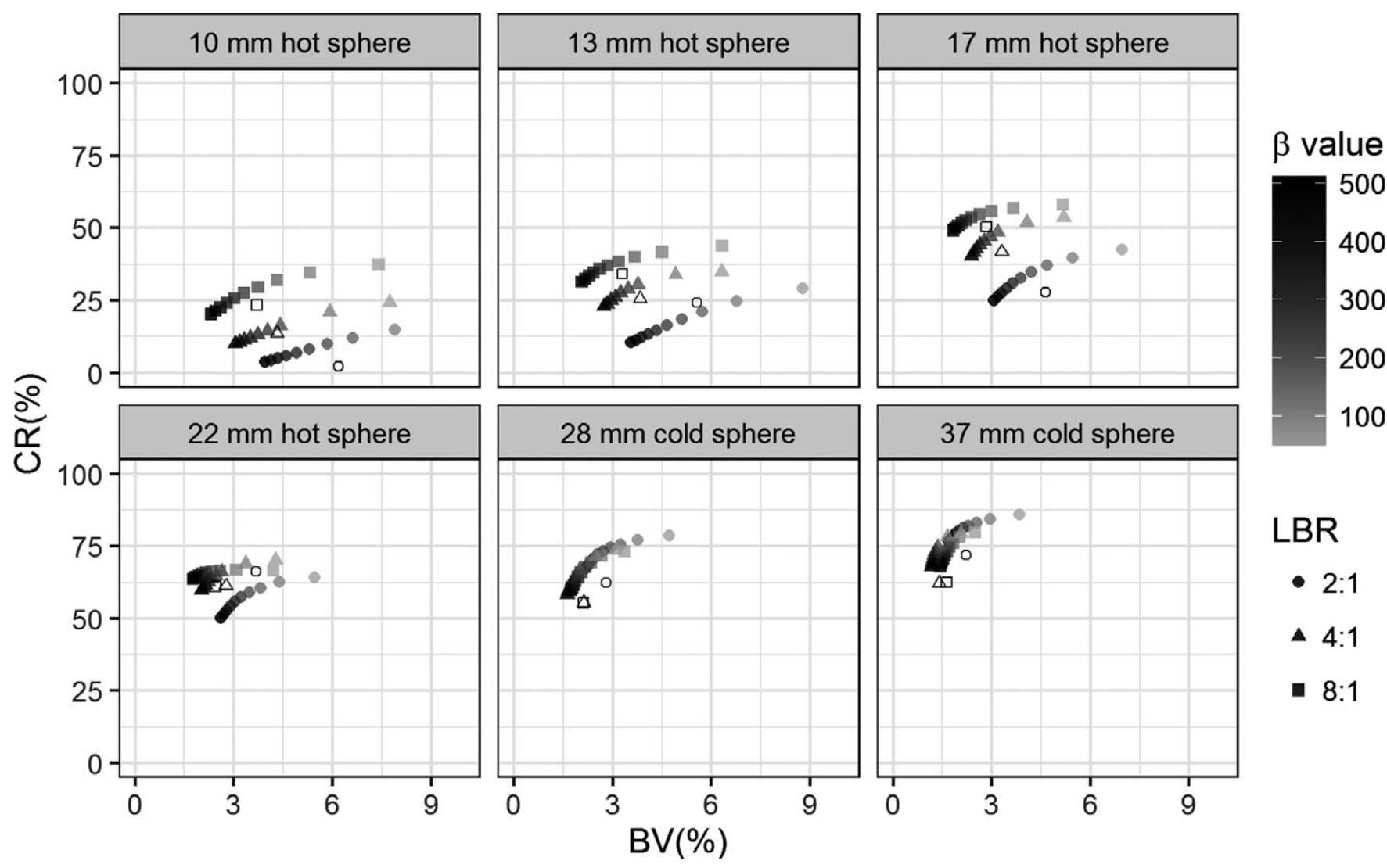

FIG. 1. Contrast Recovery vs Background Variability curves depending on the Q.Clear reconstruction $\beta$ value, the Lesion-to Background Ratio and the sphere diameter, for the NEMA image quality phantom and an acquisition time of $120 \mathrm{~s}$. White filled points represent a VPHD-S reconstruction (12 subsets, 4 iterations, and a $4.8 \mathrm{~mm}$ FWHM filtering).

Figure 4, presents a quantitative analysis for a single distinctive lesion selected in each torso study. As the $\beta$ value increased, $\mathrm{SUV}_{\max }$ decreased and lesion MTV increased [Fig. 4(d) and 4(e)]. Noise decreases rapidly as the $\beta$ value increases up to 250 , followed by a slow decrease [Fig. 4(a)]. In addition, there is a small decrease in contrast and an increase in SNR [Fig 4(b) and 4(c)]. This increase is not due to a variation in the liver $\mathrm{SUV}_{\text {mean }}$, as this parameter remained almost constant [Fig 4(f)]. For the SNR, there are two patients which maximize during the interval. These are patient 1 and 3 which, in turn, are the lowest MTV analyzed (1.3 and 1.4 $\mathrm{cm}^{3}$, respectively).

Figure S2 presents the comparison between Q.Clear and VPHD-S in terms of contrast and noise. From the point of view of contrast, VPHD-S reconstructions performed worse for all $\beta$ values, whil when considering noise, Q.Clear reconstruction outperforms VPHD-S reconstructions only for the lowest $\beta$ values.

In the evaluation of torso image quality by expert readers [Fig. 5(a)], weighted Cohen Kappa coefficients between rankers were: 0.60 for conspicuity, 0.60 for liver, 0.79 for mediastinum, 0.56 for lung, and 0.68 for the overall image quality. As a general trend, an increase in the $\beta$ value improves image quality assessment for all parameters until achieving a plateau of comparable appraisement, comprising the range of $\beta$ values between 300 and 500 . The only exceptions are bone marrow, which presents a considerable decrease after a $\beta$ of 400 , and lungs, which present a steady increase after a $\beta$ value of 350. Lesion conspicuity is the highest ranked item for Q.Clear reconstructions. In comparison, VPHD-S algorithm presents lower-ranked reconstructions than Q.Clear for $\beta$ values higher than 300 .

In the assessment of brain studies image quality [Fig. 5(b)], weighted Cohen Kappa coefficients between rankers were: 0.50 for gyri, 0.61 for basal ganglia, and 0.70 for the overall image quality. In contrast from torso acquisitions, highest ranked reconstructions are around $\beta$ values of 150 250 , decreasing the assessments as the $\beta$ increases. To illustrate these results, Fig. S3, presents the central axial profile for the brain images shown in Fig. 3, where it can be seen that the use of lower $\beta$ values implies a pronounced definition. VPHD-S presents lower ranked items than all Q.Clear reconstructions.

\section{C. Texture indices}

Table II presents the COV for each TI on all studied $\beta$ values, and for a clinical subset between 300 and 450. In the clinical range, all parameters except LZE presented COV values under 10\%. Entropy, SRE and LRE presented values under $1 \%$. When the value of $\beta$ values is extended to all the $\beta$ 

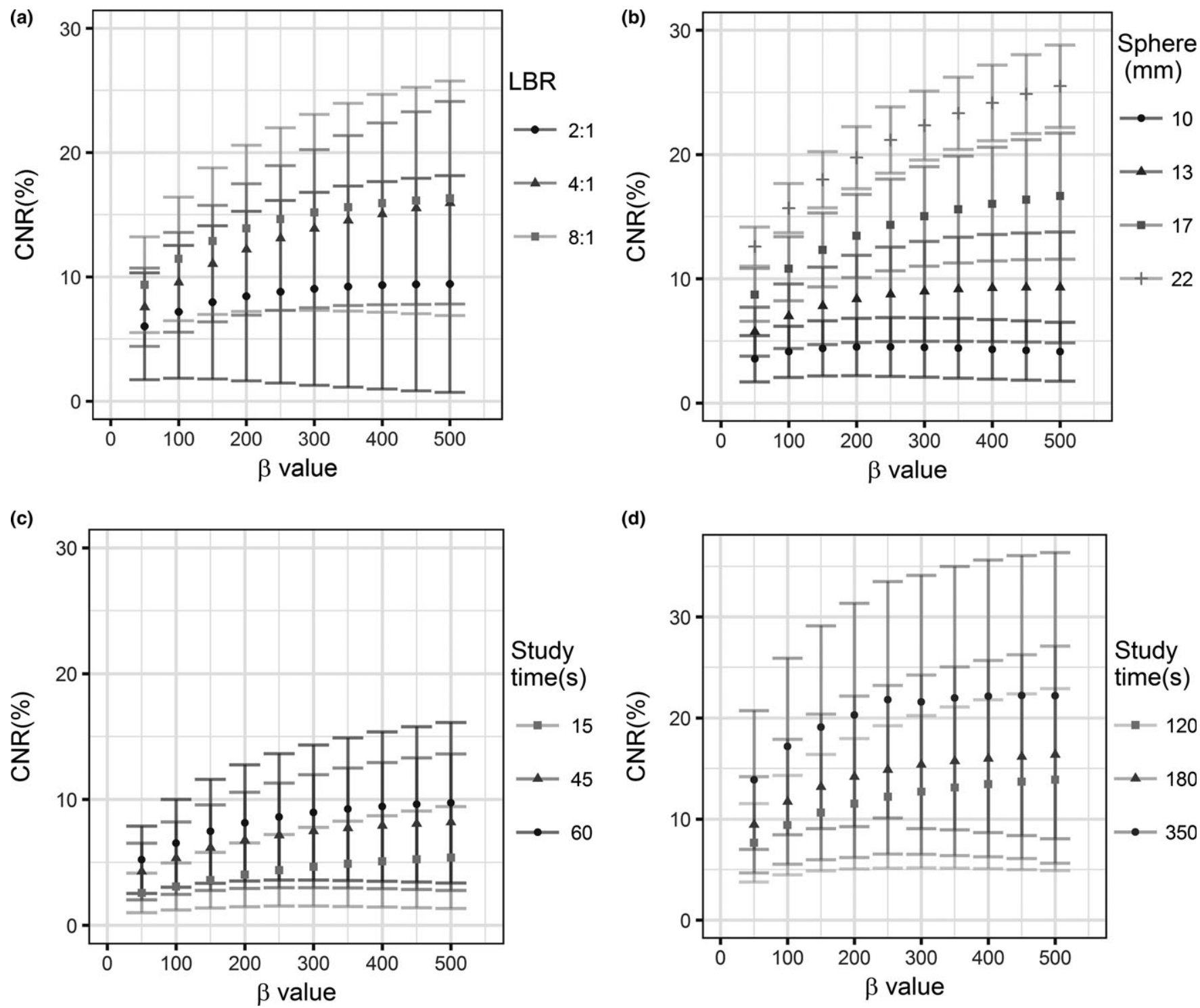

FIG. 2. Hot lesions contrast-to-noise ratio as a function of the Q.Clear reconstruction $\beta$ values (ranging from 50 to 500 ), for each studied variable averaged over the rest. Impact of (a) Lesion-to-background ratio averaged over sphere sizes and a study time of $120 \mathrm{~s}$, (b) sphere size averaged for all LBR and a study time of $120 \mathrm{~s}$, (c) and (d) study time averaged for all LBR and sphere sizes.

TABLE I. Mean $\beta$ value which maximizes the CNR, rounded to the nearest studied value, for spheres from 10 to $13 \mathrm{~mm}, 10$ to $17 \mathrm{~mm}$, and 10 to $22 \mathrm{~mm}$. Only hot spheres were considered.

\begin{tabular}{lccc}
\hline \hline & \multicolumn{3}{c}{ Spheres (mm) } \\
\cline { 2 - 4 } Time (s) & $10-13$ & $10-17$ & $10-22$ \\
\hline 15 & 350 & 400 & 400 \\
45 & 300 & 350 & 400 \\
60 & 300 & 350 & 400 \\
120 & 300 & 350 & 400 \\
180 & 300 & 350 & 400 \\
350 & 200 & 250 & 350 \\
\hline \hline
\end{tabular}

range, only Entropy, SRE, LRE, SZE, and Homogeneity presented COV values $<10 \%$, on the other hand Correlation, HGRE, and LZE presented the higher COV (21\% and 23\%, respectively). The behavior of each TI as a function of $\beta$ can be seen on Fig. S4.

\section{DISCUSSION}

As far as we know, this is the first publication on the behavior of Q.Clear algorithm, a BSREM method for reconstruction which PSF modeling and the control of noise through the use of a penalty term, in a high sensitivity BGO PET scanner; in contrast to previous studies on LYSO scanners. We have focused on different counts statistics and LBR studies, and on the optimization of different acquisition protocols and clinical applications (oncological torso and brain studies). The effect of the Q.Clear reconstruction penalty term on tumor texture metrics robustness was also evaluated, in comparison to previous studies that focused on the effect of OSEM reconstruction parameters. 
(a)

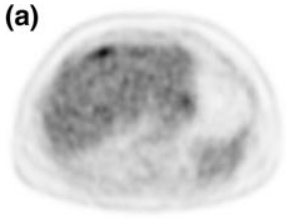

(f)

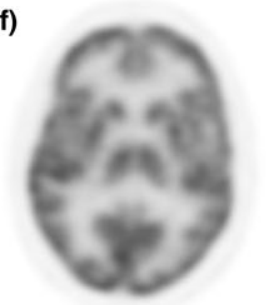

(b)

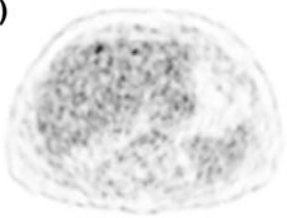

(g)

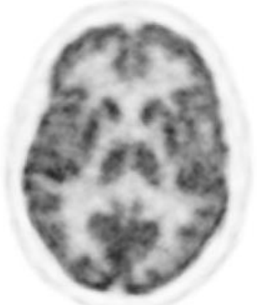

(c)

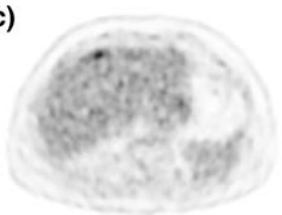

(h)

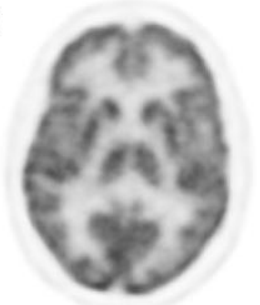

(d)

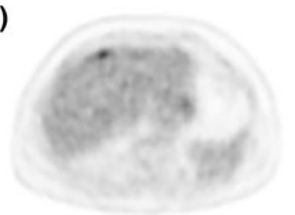

(i)

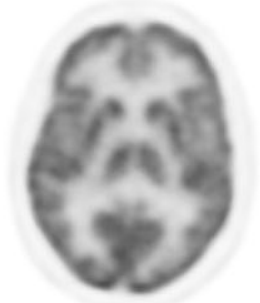

(e)

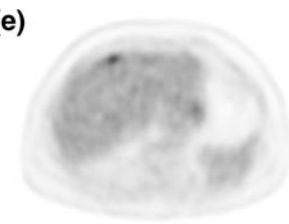

(j)

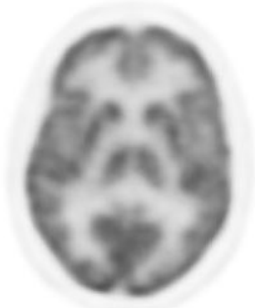

FIG. 3. Example of Q.Clear quality image dependence on $\beta$ value. Torso acquisition from a patient weighted $63 \mathrm{~kg}$ injected with $166 \mathrm{MBq}, 2.5 \mathrm{~min}$ acquisition, reconstructed using (a) VPHD-S (12 subsets, 4 iterations $4.8 \mathrm{~mm}$ FWHM filtering, SUVmax = 8.92), (b) Q.Clear $\beta 50$ (SUVmax $=15.92$ ), (c) Q.Clear $\beta 150$ $($ SUVmax $=12.53)$, (d) Q.Clear $\beta 350$ (SUVmax $=9.98)$, and (e) Q.Clear $\beta 500$ (SUVmax $=8.95)$. Brain acquisition from a patient weighted $87 \mathrm{~kg}$ injected with $294 \mathrm{MBq}, 5$ min acquisition reconstructed using (f) VPHD- S (12 subsets, 4 iterations $4.8 \mathrm{~mm}$ FWHM filtering), (g) Q.Clear $\beta$ 50, (h) Q.Clear $\beta$ 150, (i) Q.Clear $\beta 350$, and (j) Q.Clear $\beta$ 500. Pixel sizes were $2.3 \mathrm{~mm}$ for torso scans and $1.6 \mathrm{~mm}$ for brain scans (60 and $40 \mathrm{~cm}$ FOV, respectively).
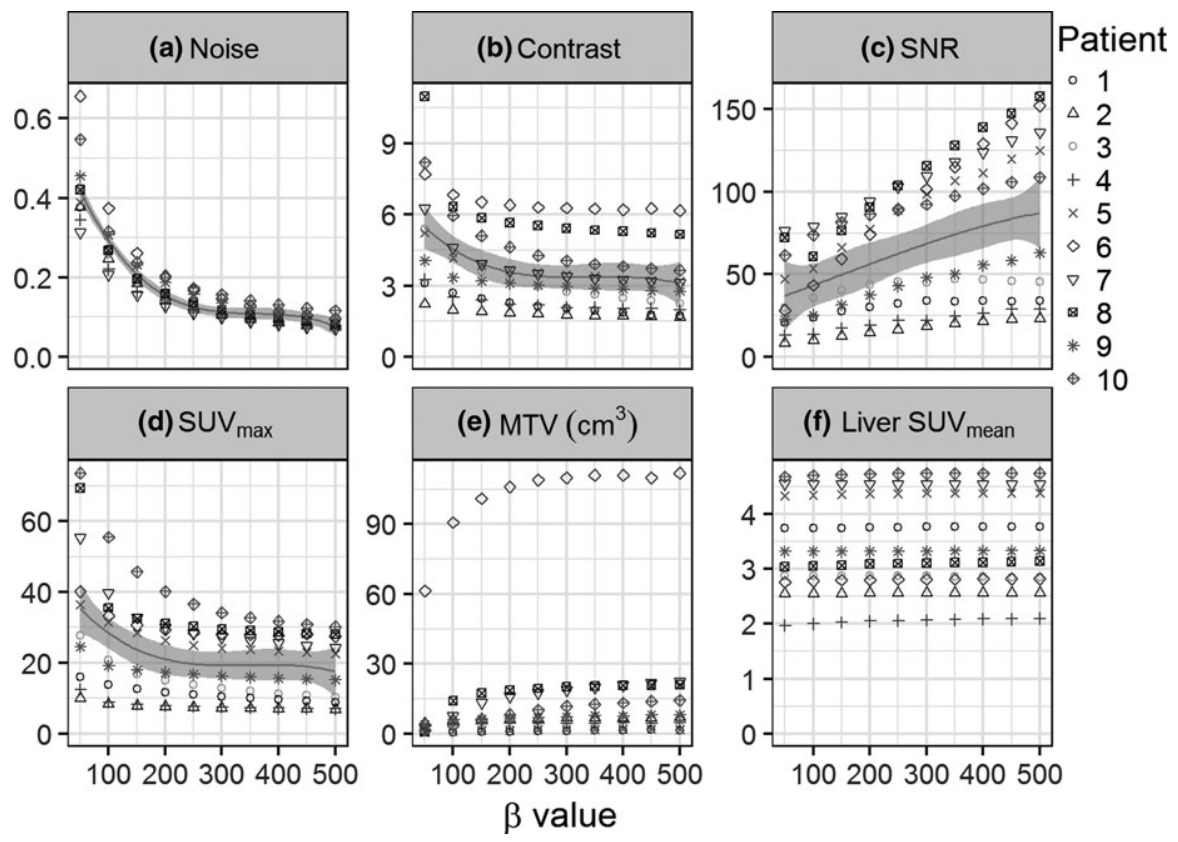

FIG. 4. (a) Noise, (b) mean contrast and (c) mean SNR, (d) liver SUVmean, (e) lesion MTV, and (f) lesion SUVmax, for each torso acquisition analyzed. Continuous lines in captions a- $\mathrm{d}$ represents smoothed conditional mean using cubic splines and corresponding confidence interval.

Clinically, OSEM reconstructions usually includes a Gaussian post-filtering between 4 and $7 \mathrm{~mm}$ FWHM in addition to the inherent smoothing produced by the combination of iterations and subsets selected (3 and 5). On the other hand, Q.Clear algorithm applies a selective filtering dependent on the surrounding activity. ${ }^{8}$ Thus, a direct comparison of both algorithms is not straightforward due to the nature of the selective filtering, which can have direct results on different anatomical zones or lesion conditions. The most direct consequence of this selective filtering is to improve lesion detectability from the VPHD-S algorithm, ${ }^{11}$ which this study validates in phantom and clinical data on a BGO scanner, from a clinical point of view. Comparable quantitative metrics (CR) could be obtained for both algorithms if there is an increase in the iterations or subsets, or a reduction of the total post-filtering in the VPHD-S algorithm. However, the increase in noise in the image will make it useless in clinical practice, similar as the use of low $\beta$ values in the Q.Clear algorithm.

Moreover, the present phantom study shows how the image quality improvement is not homogenous, depending on LBR and total administered activity, the latter simulated by different acquisition times. In general terms, as the $\beta$ increases the quantification capacity (measured as CR and 


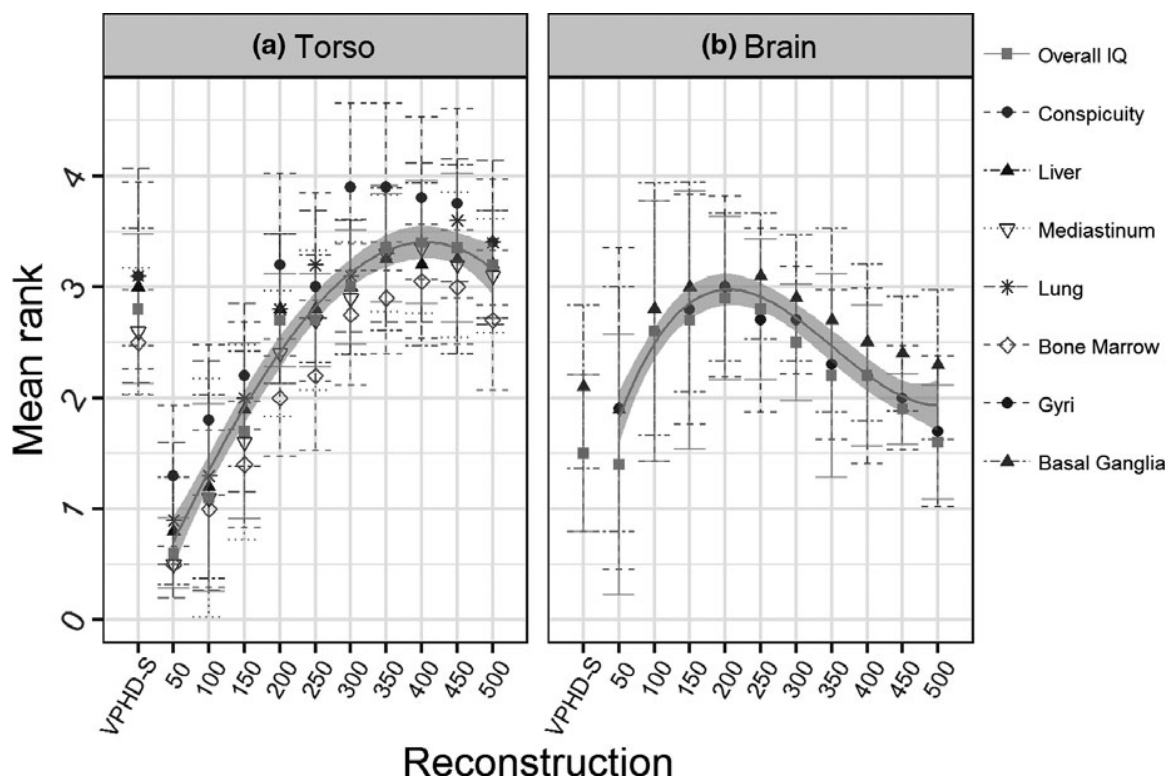

FIG. 5. Image quality assessment ranked by expert nuclear medicine physicians for (a) torso acquisitions and (b) brain studies, as a function of the $\beta$ value and different anatomical zones. Mean values with standard deviation. Continue line represents smoothed conditional mean using cubic splines and corresponding confidence interval.

TABLE II. Variability in texture indices COV over two different ranges of $\beta$ values (300-450), for phantom and patients acquisitions.

\begin{tabular}{lllll}
\hline \hline$\beta$ values & \multicolumn{1}{c}{ COV $<5 \%$} & \multicolumn{1}{c}{$5 \%<\mathrm{COV}<10 \%$} & $10 \%<\mathrm{COV}<20 \%$ & COV $>20 \%$ \\
\hline $300-450$ & SUV $_{\text {mean }}, \mathrm{SUV}_{\max }$, Homogeneity, & TLG, Correlation, LGRE, & \multirow{2}{*}{ LZE } & \\
& Entropy, SRE, LRE & HGRE, LGZE, HGZE & & \\
$100-500$ & Entropy, SRE, LRE & Homogeneity, SZE & SUV $_{\text {mean }}, \mathrm{SUV}_{\text {max }}$, TLG, LGRE & Correlation, HGRE, HGZE, LZE \\
\hline \hline
\end{tabular}

contrast) decreases, as well as the noise, leaving a total increase on detectability (in terms of CNR or SNR for phantoms or patients, respectively). Both parameters increase with the penalization factor until a plateau, which can be outside the studied range $(\beta>500)$ or can be in values as lower as 150 , depending on the acquisition time and the LBR, as shown by the phantom studies. After this plateau, some spheres or lesions present a slight decrease on CNR or SNR. These results point out that a tempting practice of increasing too much the penalized value can have a negative impact on lesion detectability. Thus, in a general purpose oncological torso scan, phantom results suggest an optimum $\beta$ value between 300 and 400 depending on the LBR, the lesion size and acquisition time (Table I). Phantom studies also show the relation between $\mathrm{BV}$, contrast recovery, LBR and the penalization factor. Although the phantom background concentrations were similar in the three LBR experiments, a difference up to $1 \%-2 \%$ was found in the BV. This has also been found by others publications. ${ }^{23-25}$

The smallest lesions are the most challenging, thus it would be of interest to obtain a general $\beta$ to optimize the reconstruction based on an increase in the small lesions detectability. Therefore, one should consider a $\beta$ value which maximizes the CNR for the 10 and $17 \mathrm{~mm}$ spheres, assuming a generic LBR. Using this criterion, the suggested optimal values should be around 350 for a 60-120 s acquisition and a $5.5 \mathrm{kBq} / \mathrm{cm}^{3}$ concentration, which simulates a torso acquisition. Although the role of phantom data is to shown trends in a well-controlled situation, phantom findings are in agreement with the image quality assessment of the torso reconstructions, with a $\beta$ value of 350 for studies acquired with the same statistics (product of the acquisition time and activity concentration of $120 \mathrm{~s} \times 2.5 \mathrm{kBq} / \mathrm{g}$ ). The present results for torso acquisitions are similar to previous studies using a LYSO PET, where a $\beta$ value of 400 is recommended as a general practice, ${ }^{11}$ although they only considered one LBR and a single acquisition time. In the case of brain acquisitions, which imply higher concentrations, the $\beta$ value should be around 200 .

This criterion to obtain an optimal value has its limitations, as there is not a general way to weight the importance of each sphere or LBR, and also the maximum value in the CNR is highly dependent in the measurement uncertainty. Despite this, it presents the general trends on the behavior of the Q.Clear algorithm. Similar limitations were found by others when recommendations have been done for an OSEM with TOF reconstruction, providing values of iterations and subsets numbers, and post smoothing filter. ${ }^{17,26,27}$

As previously shown, the desired range of $\beta$ values in torso studies remains in an interval between 300 and 400, and 
so the exact choice of the desired $\beta$ could be performed in terms of other factors. Although a defined $\beta$ should be established for oncological ${ }^{18} \mathrm{~F}$-FDG torso acquisitions in order to preserve traceability and comparability of SUV values, of especial interest in patient follow-up and in clinical trials, this study suggests that a good practice could be to perform different reconstructions for different anatomical zones or goals. Additionally, more work should be done on this topic to know if a specific tuning of the penalized factor could be translated in an increase in specificity or sensitivity in clinical situations. One general concern of Bayesian reconstruction algorithms is the reconstruction time, which could limit the practical viability of performing different reconstructions for the same patient. In the case of the Q.Clear implantation, reconstruction time depends on the hardware provided by the manufacturer; for images in this study it is approximately 1 min per bed.

Results also open the question of the Q.Clear reconstruction optimization for each different radiopharmaceutical and clinical application. For example, it was recently reported that for ${ }^{90} \mathrm{Y}$ TOF PET imaging a $\beta$ value of 4000 is the recommended choice, ${ }^{28}$ far more elevated than the values considered in the present work. This extreme case is due to the low statistics of ${ }^{90} \mathrm{Y}$ PET imaging, ${ }^{29}$ so different results than those presented in this study can be achieved for other nuclides or pharmaceuticals.

The benefit of using different optimization parameters on different anatomical areas, where count statistics and resolution demands are different, can be clearly seen on brain images, where the required $\beta$ values are much lower than the one that could be considered clinically acceptable on a torso acquisition. As a general rule, as the statistics of the image increase, the $\beta$ can be decreased. For brain studies, the FOV reduction extracts counts of the sinogram from outside the brain, avoiding some scatter and random events to be corrected during the reconstruction. One should also consider that the Q.Clear reconstruction has a FOV dependence, ${ }^{13}$ as its variation could slightly modify our results in different anatomical regions. Moreover, differences in pixel sizes and FOV for brain and torso scans influence the optimization parameters and should be considered if the results of this study are going to be extrapolated to other acquisition conditions.

Regarding TI, its behavior is coherent with the effect that an increase in the $\beta$ value tends to homogenize the lesion; as homogeneity and LGRE increase, and Contrasts and HGRE decrease. COV Surprisingly, entropy does not present a clear pattern, which could be due to the selected resampling procedure or VOI definition. ${ }^{26}$

It is difficult to perform a direct comparison of COV from different studies, as there are some differences in methodology which could have a great impact in texture calculation, ${ }^{30}$ despite this for the proposed clinical range of $\beta$ values, we could consider that the COV obtained are compatible with the results of previous publications on the impact of reconstruction settings for OSEM-like algorithms. ${ }^{22,31}$ The interpretation of the differences in our results and previous works is not straightforward for most parameters. For example, although not a texture parameter $\mathrm{SUV}_{\max }$ seems to be a little more stable in our work, which is coherent with the results found with phantom data, and could impact the variance in other more complex parameters as LGRE or GHRE which also seems to be more stable in the clinical range of $\beta$ values. All this stability is lost when a larger set of $\beta$ values are considered, and only Entropy, SRE and LRE still presents a COV's fewer than $5 \%$.

GLRM parameters SRE, LRE, and entropy were the most robust parameters, though all of them presented a COV below $10 \%$, with the exception of LZE. Therefore, the decision of the robustness or utility of each TI should be performed in terms of other means and, as a consequence, the modification of the $\beta$ values in the interval of recommended clinical values should not have a high impact on TI.

In short, protocol optimization on Q.Clear could depend on various factors: patient anatomy, administered activity, acquisition time, acquisition zone and the user inputs in the reconstruction, as matrix size and FOV.

\section{CONCLUSION}

Q.Clear, compared with a standard OSEM reconstructions, increases $\mathrm{CR}$ and decreases $\mathrm{BV}$, producing and overall increase in CNR in phantom studies, and it increases lesions SNR in patient studies. Image quality assessment of torso and brain studies also improves. The improvement depends on the studied conditions and the penalization factor. A $\beta$ value of 350 and 200 appears to be the optimal value for ${ }^{18} \mathrm{~F}-\mathrm{FDG}$ oncology and brain PET/CT using the Q.Clear reconstruction algorithm in a BGO scanner. For torso scans, this value assures a compromise in image quality and quantitation accuracy. Texture indices show the effect of lesion homogeneity as $\beta$ value increases.

\section{CONFLICTS OF INTEREST}

Gabriel Reynés-Llompart recived research funding from General Electric Spain. All other authors declare no conflict of interests.

\footnotetext{
a) Author to whom correspondence should be addressed. Electronic mail: gabriel.reynes@idi.gencat.cat; Telephone: 932640036.
}

\section{REFERENCES}

1. Gámez-Cenzano C, Pino-Sorroche F. Standardization and quantification in FDG-PET/CT imaging for staging and restaging of malignant disease. PET Clin. 2014;9:117-127.

2. Brianzoni E, Rossi G, Ancidei S, et al. Radiotherapy planning: PET/CT scanner performances in the definition of gross tumour volume and clinical target volume. Eur J Nucl Med Mol Imaging. 2005;32:1392-1399.

3. Boellaard R, Delgado-Bolton R, Oyen WJ, et al. FDG PET/CT: EANM procedure guidelines for tumour imaging: version 2.0. Eur J Nucl Med Mol Imaging. 2015;42:328-354.

4. Nuyts J, Michel C, Brepoels L, et al. Performance of MAP reconstruction for hot lesion detection in whole-body PET/CT: an evaluation with 
human and numerical observers. IEEE Trans Med Imaging. 2009;28:67-73.

5. Qi J, Leahy RM. Iterative reconstruction techniques in emission computed tomography. Phys Med Biol. 2006;51:R541.

6. Jaskowiak CJ, Bianco JA, Perlman SB, Fine JP. Influence of Reconstruction Iterations on 18F-FDG PET/CT Standardized Uptake Values. J Nucl Med. 2005;46:424-428.

7. Brendle C, Kupferschläger J, Nikolaou K, La Fougère C, Gatidis S, Pfannenberg C. Is the standard uptake value (SUV) appropriate for quantification in clinical PET imaging? - Variability induced by different SUV measurements and varying reconstruction methods. Eur J Radiol. 2015;84:158-162.

8. Ahn S, Fessler JA. Globally convergent image reconstruction for emission tomography using relaxed ordered subsets algorithms. IEEE Trans Med Imaging. 2003;22:613-626.

9. Ahn S, Ross SG, Asma E, et al. Quantitative comparison of OSEM and penalized likelihood image reconstruction using relative difference penalties for clinical PET. Phys Med Biol. 2015;60:5733-5751.

10. Nuyts J, Fessler JA. A penalized-likelihood image reconstruction method for emission tomography, compared to postsmoothed maximum-likelihood with matched spatial resolution. IEEE Trans Med Imaging. 2003;22:1042-1052.

11. Teoh EJ, McGowan DR, Macpherson RE, Bradley KM, Gleeson FV. Phantom and clinical evaluation of the bayesian penalized likelihood reconstruction algorithm Q.Clear on an LYSO PET/CT system. $J$ Nucl Med. 2015;56:1447-1452.

12. Parvizi N, Franklin JM, McGowan DR, Teoh EJ, Bradley KM, Gleeson FV. Does a novel penalized likelihood reconstruction of 18F-FDG PETCT improve signal-to-background in colorectal liver metastases? Eur $J$ Radiol. 2015;84:1873-1878.

13. Asma E, Ahn S, Ross SG, Chen A, Manjeshwar RM. Accurate and consistent lesion quantitation with clinically acceptable penalized likelihood images. IEEE Nuclear Science Symposium Conference Record. 2012;4062-4066.

14. Slomka PJ, Pan T, Germano G. Recent advances and future progress in PET instrumentation. Semin Nucl Med. 2015;46:5-19.

15. Surti S, Karp JS. Advances in time-of-flight PET. Phys Med. 2016;32:12-22.

16. Teoh EJ, McGowan DR, Bradley KM, Belcher E, Black E, Gleeson FV. Novel penalised likelihood reconstruction of PET in the assessment of histologically verified small pulmonary nodules. Eur Radiol. 2016;26:576-584.

17. Carlier T, Bailly C. State-Of-The-Art and recent advances in quantification for therapeutic follow-up in oncology using PET. Front Med. 2015;2:1-12.

18. Reynés-Llompart G, Gámez-Cenzano C, Romero-Zayas I, RodríguezBel L, Vercher-Conejero JL, Martí-Climent JM. Performance characteristics of the whole-body discovery IQ PET/CT system. J Nucl Med. 2017;58:1155-1161.

19. National Electrical Manufacturers Association. Performance measurements of positron emission tomographs. Rosslyn, USA: NEMA Standards Publication NU 2-2012; 2012).

20. Lois C, Jakoby BW, Long MJ, et al. An assessment of the impact of incorporating time-of-flight information into clinical PET/CT imaging. J Nucl Med. 2010;51:237-245.

21. Orlhac F, Soussan M, Maisonobe J-A, Garcia CA, Vanderlinden B, Buvat I. Tumor texture analysis in 18F-FDG PET: relationships between texture parameters, histogram indices, standardized uptake values, metabolic volumes, and total lesion glycolysis. J Nucl Med. 2014;55:414-422.

22. Yan J, Chu-Shern JL, Loi HY, et al. Impact of image reconstruction settings on texture features in 18F-FDG PET. J Nucl Med. 2015;56:16671673.

23. Teräs M, Tolvanen T, Johansson JJ, Williams JJ, Knuuti J. Performance of the new generation of whole-body PET/CT scanners: Discovery STE and Discovery VCT. Eur J Nucl Med Mol Imaging. 2007;34:1683-1692.
24. Jakoby BW, Bercier Y, Watson CC, Bendriem B, Townsend DW. Performance characteristics of a new LSO PET/CT scanner with extended axial field-of-view and PSF reconstruction. IEEE Trans Nucl Sci. 2009;56:633-639.

25. Rausch I, Cal-González J, Dapra D, et al. Performance evaluation of the Biograph mCT Flow PET/CT system according to the NEMA NU22012 standard. EJNMMI Phys. 2015;2:26.

26. Orlhac F, Nioche C, Soussan M, Buvat I. Understanding changes in tumor texture indices in PET: a comparison between visual assessment and index values in simulated and patient data. $J$ Nucl Med. 2017;58:387-392.

27. Prieto E, Domínguez-Prado I, García-Velloso MJ, Peñuelas I, Richter JÁ, Martí-Climent JM. Impact of time-of-flight and point-spread-function in SUV quantification for oncological PET. Clin Nucl Med. 2013;38:103-109.

28. Rowley LM, Bradley KM, Boardman P, Hallam A, McGowan DR. Optimization of Image Reconstruction for Yttrium-90 SIRT on a LYSO PET/ CT System Using a Bayesian Penalized Likelihood Reconstruction Algorithm. Med: J. Nucl; 2016.

29. Martí-Climent JM, Prieto E, Elosúa C, et al. PET optimization for improved assessment and accurate quantification of ${ }^{90}$ Y-microsphere biodistribution after radioembolization. Med Phys. 2014;41:92503.

30. Hatt M, Tixier F, Pierce L, Kinahan PE, Le Rest CC, Visvikis D. Characterization of PET/CT images using texture analysis: the past, the present. . . any future? Eur J Nucl Med Mol Imaging. 2016;44:1-15.

31. Shiri I, Rahmim A, Ghaffarian P, Geramifar P, Abdollahi H, BitarafanRajabi A. The impact of image reconstruction settings on 18F-FDG PET radiomic features: multi-scanner phantom and patient studies. Eur Radiol. 2017;27:1-12.

\section{SUPPORTING INFORMATION}

Additional supporting information may be found online in the Supporting Information section at the end of the article.

Data S1. Supplemental material.

Fig. S1. Cold lesions contrast-to-noise ratio as a function of the Q.Clear reconstruction $\beta$ values (ranging from 50 to 500 ), lesion-to-background ratio and sphere diameter, plotted over different acquisition times.

Fig. S2. Mean lesion contrast vs liver noise for each patient and each $\beta$ value. Points surrounded by a diamond correspond to VPHD-S points.

Fig. S3. Central axial profile for a brain scan from a patient weighted $87 \mathrm{~kg}$ injected with $294 \mathrm{MBq}, 5$ min acquisition and reconstructed using VPHD-S (8 subsets, 4 iterations $4.8 \mathrm{~mm}$ FWHM filtering), and Q.Clear with $\beta$ values of 50, 150,350 , and 500 .

Fig. S4. Textural Indices for each lesion analyzed as a function of $\beta$ value. Continue line represents smoothed conditional mean using cubic splines and corresponding confidence interval.

Table S1. Using a LBR 2:1, the $\beta$ value which maximize de CNR For each sphere size (in $\mathrm{mm}$ ) and acquisition time.

Table S2. Using a LBR 4:1, the $\beta$ value which maximize de CNR For each sphere size (in $\mathrm{mm}$ ) and acquisition time.

Table S3. Using a LBR 8:1, the $\beta$ value which maximize de CNR For each sphere size (in $\mathrm{mm}$ ) and acquisition time.

Table S4. Characteristics of all lesions analyzed. SUV values are taken from a $\beta$ of 300 . 